\title{
Developing Acceptance Sampling Plans based on Incapability Index $C_{p p}$
}

\author{
Liang-Chyau Sheu ${ }^{1, *}$, Chi-Huang Yeh ${ }^{2, *}$, Ching-Ho Yen ${ }^{3}$ and Chia-Hao Chang ${ }^{4}$ \\ ${ }^{1}$ Department of Technology Management, Chung Hua University, Hsinchu, Taiwan \\ ${ }^{2} \mathrm{Ph}$. D. Program of Technology Management, Chung Hua University, Hsinchu, Taiwan \\ ${ }^{3}$ Department of Industrial Engineering \& Management Information, Huafan University, Taipei, Taiwan \\ ${ }^{4}$ Department of Nursing, Chang Gung University of Science and Technology, Chiayi Campus, Chiayi, Taiwan
}

Received: 20 Sep. 2013, Revised: 18 Dec. 2013, Accepted: 19 Dec. 2013

Published online: 1 Sep. 2014

\begin{abstract}
Most acceptancesampling plans focus on the percentage of defective products instead of considering the process loss, which doesn't distinguish among the products that fall within the specification limits. Therefore, it is essential to develop an acceptance sampling plan with process loss consideration. In this paper, a variable sampling plan based on incapability index $C_{p p}$ is proposed to deal with lot sentencing. The required sample sizesn and the critical acceptance value $c$ with some combination of acceptance quality level are tabulated. One example is used to illustrate the proposed methodology. The proposed sampling plan provides a feasible policy, which can be applied to products requiring low process loss where classical sampling plans cannot be applied.
\end{abstract}

Keywords: acceptance sampling plan,process loss,incapability index, lot sentencing, critical acceptance values.

\section{Introduction}

In the area of quality control, acceptance sampling plan is a very widely used method of statistical quality control. Acceptance sampling is the judgment of one lot based on the information obtained from the samples. However, acceptance sampling plans have two unavoidable risks, rejecting good lots and accepting bad lots.An acceptance sampling plan virtually consists of a sample size and an acceptance or rejection criterion. A well-designed sampling plan can significantly reduce the difference between the expected and the actual supplied product quality. The performance of an acceptance sampling plan is based on the operating characteristic (OC) curve quantifying the risks for consumers and producers.The OC curve plots the probability of accepting the lot against actual product fraction defective, which displays the discriminatory power of the sampling plan. That is, the OC curve shows the probability of accepting a product lot in terms of the product fraction defective (nonconformities), which provides the producer and the buyer a common ground for judging whether the sampling plan is appropriate.
The fundamental concepts of variable sampling plans were introduced by Jennett and Welch [1939]. Lieberman and Resnikoff [1955] evolved generous tables and OC curves for various AQLs for MIL-STD-414 sampling plan. Das and Mitra [1964] examined the effect of non-normality on the performance of the sampling plans. Owen [1967) developed sampling plans for various levels of probabilities of Type I error when the standard deviation is unknown under the normal distribution. Guenther [1969] developed a systematic search procedure, which provided some published tables of binomial, hyper-geometric, and Poisson distributions to gain the desired acceptance sampling plans. Kao [1971] provided the comparison between the attribute acceptance sampling plans and the variable acceptance sampling plans.Stephens [1978) used a normal approximation to the binomial distribution to give a closed form solution for single sample acceptance sampling plans. Hailey [1980] provided a computer program to acquire single sampling plans with minimum sample size based on either the binomial or Poisson distribution. Hald[1981] made a systematic discussion of the existing statistical theory of lot-by-lot sampling inspection by attributes and

\footnotetext{
*Corresponding author e-mail: 1lcsheu@ chu.edu.tw, d09703016@chu.edu.tw
} 
offered some tables for them.Govindaraju and Soundararajan [1986] built variables sampling plans that mate the OC curves of MIL-STD-105D. Suresh and Ramanathan [1997] developed a sampling plan based on a more general symmetric family of distributions.

Since the above variable acceptance sampling plans do not consider the viewpoint that the quality between products falling within the specification limits may be different, some authors have designed the variable acceptance sampling plans using process capability indices as below. Pearn and $\mathrm{Wu}$ [2006] proposed a variable sampling plan based on $C_{p l}$ and $C_{p u}$. Pearn and $\mathrm{Wu}$ [2006] used the index $C_{p m}$ to design a variable sampling plan. Pearn and $\mathrm{Wu}$ [2007] developed a decision making method for product based on $C_{p k}$. Wu and Pearn [2008] offered a variable sampling plan based on $C_{p m k}$ for product acceptance determination. Yen and Chang [2009] used the process loss index $L_{e}$ to design a variable sampling plan.In this paper, we develop a variables sampling plan using the incapability index $C_{p p}$ to deal with lot sentencing problem for processes requiring low process loss. The rest of paper is organized as follows. Section 2 describes the process capability indices. In Section 3 we present the design of variable sampling plan based on $C_{p p}$. In Section 4, a numerical example is used to illustrate the proposed methodology. Finally, conclusions are made in Section 5.

\section{Process Capability Indices}

The capability indices are widely used in the manufacturing industry to evaluate process performance. As we know, process capability indices and related analysis have been studied and have been the focus in quality assurance and capability analysis in recent years. Those indices establish the relationship between the actual process performance and the manufacturing specifications. When a process is in a good state of statistical control, its process capability index will be large. On the contrary, it will be small when a process is out of control. The most commonly used capability indices $C_{p}$ and $C_{p k}$ (Kane[1986]) are defined as follows:

$$
C_{p}=\frac{U S L-L S L}{6 \sigma}, C_{p K}=\left\{\frac{U S L-\mu}{3 \sigma}, \frac{\mu-L S L}{3 \sigma}\right\},
$$

where USL and $L S L$ are the upper and lower specification limits, respectively, $\mu$ is the process mean, and $\sigma$ is the process standard deviation.

The index $C_{p}$ measures the overall process variation relative to the specification tolerance. The index $C_{p k}$ takes into account the magnitude of process variation as well as the degree of process centering. However, these two indices fail to account for the process centering. Chan et al. [1988] proposed a new capability index, called $C_{p m}$, to evaluate the capability of process while taking into account the process centering. The index $C_{p m}$ is defined as follows:

$$
C_{p m}=\frac{U S L-L S L}{6 \sqrt{\sigma^{2}+(\mu-T)^{2}}},
$$

where $U S L$ and $L S L$ are the upper and lower specification limits, respectively, $T$ is the target value, $\mu$ is the process mean, and $\sigma$ is the process standard deviation. This index emphasizes on measuring the ability of process to cluster around the target, which reflects the degrees of process targeting.

\subsection{Incapability index $C_{p p}$}

Since the statistical properties of the capability index $C_{p m}$ is analytically intractable, Greenwich and Jahr-Schaffrath (1995) proposed another capability index $C_{p p}$, called incapability index, to evaluate the process performance. The incapability index $C_{p p}$ can be expressed as $C_{p p}=C_{i a}+C_{i p}$, where $C_{i a}$ (inaccuracy index) and $C_{i p}$ (imprecision index) are used to measure the process accuracy and the process precision, respectively. The incapability index $C_{p p}$ is defined as

$$
C_{p p}=\left(\frac{\mu-T}{D}\right)^{2}+\left(\frac{\sigma}{D}\right)^{2}
$$

where $\mu$ is the process mean, $\sigma$ is the process standard deviation, $T$ is the target value, $D=d / 3$, $d=(U S L-L S L) / 2$ is the half specification width, $U S L$ and $L S L$ are the upper and the lower specification limits, $C_{i a}=(\mu-T) / D^{2}$, and $C_{i p}=\sigma^{2} / D^{2}$. The index $C_{p p}$ is a simple transformation of the index $C_{p m}$, which provides an uncontaminated separation between information concerning the process accuracy and process precision. From the above definition, it is noted that the mathematical relationship, $C_{p p}=\left(C_{p m}\right)^{-2}$, $C_{i a}=\left(3-3 C_{a}\right)^{2}\left(C_{a}=1-|\mu-T| / d\right)$ and $C_{i p}=\left(C_{p}\right)^{-2}$ can be constructed. The advantage of $C_{p p}$ over $C_{p m}$ is that the estimator of the former has better statistical properties than that of the latter, as the former does not involve a reciprocal transformation of process mean and variance. The above indices $C_{p k}, C_{p m}$ can provide only a lower bound estimation on the process yield yield $\geq 2 \Phi(3 \times$ index value)-1. Note that the highest value that a process yield might be would not be concerned. For example, if the index value is $C$, then the yield of the process would be equal to or greater than $2 \Phi(3 C)-1$. Since the index $C_{p p}$ can be expressed as $\left(C_{p m}\right)^{-2}$, the index $C_{p p}$ can provide a lower bound estimation on the process yield (yield $\geq 2 \Phi\left(3 \sqrt{1 / C_{p p}}\right)-1$ ). Some commonly used values for $C_{p p}$ and the corresponding $C_{p m}$ values are listed in Table 1. 
Table 1: Some commonly used $C_{p p}$ and the corresponding $C_{p m}$ values

\begin{tabular}{|ccc|}
\hline Condition & $C_{p p}$ & $C_{p m}$ \\
\hline Incapable & 4 & 0.50 \\
Capable & 1 & 1.00 \\
Satisfactory & 0.5653 & 1.33 \\
Good & 0.4444 & 1.50 \\
Excellent & 0.3586 & 1.67 \\
Super & 0.25 & 2.00 \\
\hline
\end{tabular}

\subsection{Sampling Distribution of $\hat{C}_{p p}$}

In practice, sample data must be collected in order to estimate the true process capability $C_{p p}$. For measuring the process capability based on incapability index $C_{p p}$, we consider the natural estimator $\hat{C}_{p p}$, the maximum likelihood estimator (MLE) of $C_{p p}$, defined as the following:

$\widehat{C}_{P P}=\frac{(\bar{X}-T)^{2}}{D^{2}}+\frac{S^{2} n}{D^{2}}=\frac{(\bar{X}-T)^{2}}{D^{2}}+\frac{1}{n} \sum_{i=1}^{n}{\frac{\left(X_{i}-\bar{X}\right)^{2}}{D^{2}}}^{2}=\frac{\sum_{i=1}^{n}\left(X_{i}-T\right)^{2}}{n D^{2}}$

where $\quad \bar{X}=\sum_{i=1}^{n} X_{i} / n, S_{n}^{2}=\sum_{i=1}^{n}\left(X_{i}-\bar{X}\right)^{2} / n$.

According to the above equation (1) and the assumption of normal distribution, a mathematical relationship can be expressed as follows $\frac{\widehat{C}_{P P}}{C_{P P}}=\frac{\sum_{i=1}^{n}\left(X_{i}-T\right)^{2}}{n D^{2}} \times \frac{D^{2}}{\left[\sigma^{2}+(\mu-T)^{2}\right]}=\frac{\sum_{i=1}^{n}\left(X_{i}-T\right)^{2}}{\sigma^{2}}=\frac{\chi^{2} n, \delta}{n+\delta}$ where $\delta=n \zeta^{2}=n(\mu-T)^{2} / \sigma^{2}$. Thus, $\widehat{C}_{P P}$ is distributed as $C_{P P} \chi_{n, \delta}^{2} /(n+\delta)$.

\section{The Variable Sampling Plan Based on $C_{P P}$}

A well-designed sampling plan must provide a probability of at least $1-\alpha$ of accepting a lot if the lot fraction of defectives is at the contracted $A Q L$. The sampling plan must also provide a probability of acceptance no more than $\beta$ if the lot fraction of defectives is at the LTPD level, the designated undesired level preset by the buyer. Thus, the acceptance sampling plan must have its OC curve passing through those two designated points $(A Q L, 1-\alpha)$ and $(L T P D, \beta)$. To determine whether a given process is capable, we can first consider the following testing hypothesis

$$
\begin{aligned}
& H_{0}: p=A Q L \quad(\text { process is capable }) \\
& H_{1}: p=L T P D \text { (process is not capable) }
\end{aligned}
$$

To construct a variable acceptance sampling plan based on the capability index $C_{P P}$, the above hypotheses is equivalent to test

$$
\begin{aligned}
& H_{0}: C_{P P} \leqslant C_{A Q L} \\
& H_{1}: C_{P P} \leqslant C_{L T P D}
\end{aligned}
$$

Therefore, the required inspection sample size $\mathrm{n}$ and critical acceptance value $c$ of $\widehat{C}_{P P}$ for the sampling plans can be obtained by solving the following two nonlinear simultaneous equations (2) and (3).

$$
\begin{gathered}
P\left(\chi_{n, n\left(1+\zeta^{2}\right)}^{2}<\frac{n\left(1+\zeta^{2}\right) c}{C_{A Q L}}\right) \geqslant 1-\alpha \\
P\left(\chi_{n, n\left(1+\zeta^{2}\right)}^{2}<\frac{n\left(1+\zeta^{2}\right) c}{C_{L T P D}}\right) \leqslant \beta
\end{gathered}
$$

where $C_{A Q L}<C_{L T P D}$. We note that the required sample size $n$ is the smallest possible value of $n$ satisfying equations (2) and (3), and determining the $[n]$ as sample size, where $[n]$ means the least integer greater than or equal to $n$.

In fact, the parameter $\xi=(\mu-T) / \sigma$ is an unknown value, if we use $\hat{\xi}=(\bar{X}-T) / S$ to estimate the values of $\xi$, the hypothesis testing would be less reliable. To eliminate the need for further estimating the parameter $\xi$, we examine the behavior of the critical acceptance values $c$ and the sample size $n$ against the parameter $\xi$. We perform extensive calculations to obtain the critical acceptance value $\mathrm{c}$ and the sample size $n$ for $\xi^{2}=0(0.01) 9.00$, with various parameters. Figure 1 displays the surface plot for the required sample size $n$, critical acceptance value $c$, versus $\xi^{2}$ value for $C_{A Q L}=0.5917, C_{L T P D}=1.0$ with $\alpha=0.05, \beta=0.10$. From Figure 1, we can observe that the critical acceptance value will be smallest at $\xi^{2}=0$ for a specified $n$. Figure 2 plots the required sample size $\mathrm{n}$ versus $\xi^{2}$ value for $C_{A Q L}=0.8264, \quad 0.6944,0.5917,0.5102,0.4444$, $C_{L T P D}=1.0$ with $\alpha=0.05, \beta=0.10$. From Figure 2, we know that the $n$ is decreasing in $\xi^{2}$ and reaches its maximum at $\xi^{2}=0$ in all cases. To integrate the analysis from this two Figures, we can conclude that the $(n, c)$ is the most conservative at $\xi=0$ for various combination of $C_{A Q L}, C_{L T P D}$ with $\alpha$ and $\beta$. For assuring the quality of products, the most conservative $(n, c)$ can be regarded as the optimal $(\mathrm{n}, \mathrm{c})$. Hence, for practical purpose we may solve equations with $\xi=0$ to obtain the criterion of $\hat{C}_{p p}$ and the required sample size $n$, without having to estimate the parameter $\xi$.

For practical applications purpose, we calculate and tabulate the critical acceptance values and required sample sizes for the sampling plans, with commonly used $\alpha, \beta, C_{A Q L}$ and $C_{L T P D}$. Table 2 display $(n, c)$ values for producer's $\alpha$-risk $=0.01,0.025(0.025) 0.10$ and buyer's $\beta$ -risk $=0.01,0.025(0.025) 0.10$, with some quality levels, $\left(C_{A Q L}, \quad C_{L T P D}\right)=(0.5917,1.0), \quad(0.4444,0.5917)$, $(0.3673,0.4444),(0.2500,0.3673)$. For the proposed sampling plan to be practical and convenience to use, aacceptance sampling plan based on $C_{p p}$, stated as 


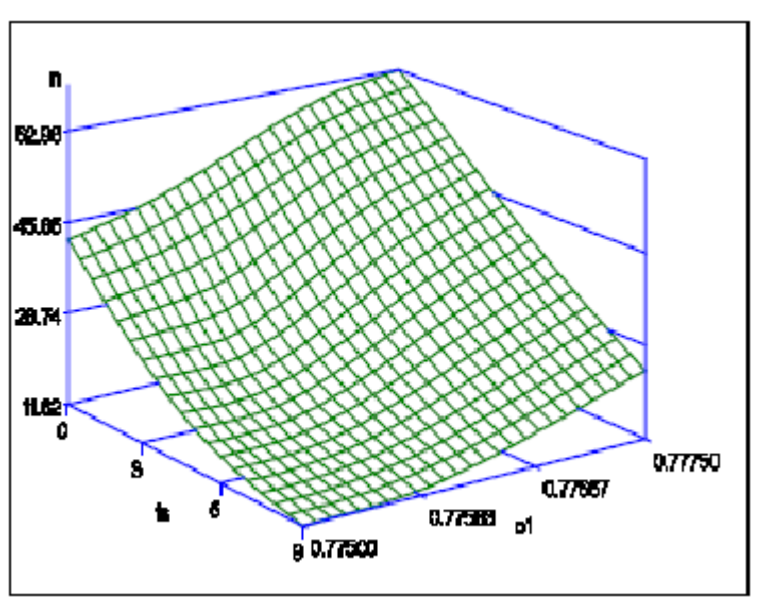

Fig. 1: The surface plot for the required sample size $n$ and critical acceptance value $c$, versus $\xi^{2}$ value for $C_{A Q L}=0.5917, C_{L T P D}=$ 1.0 with $\alpha=0.05, \beta=0.10$.

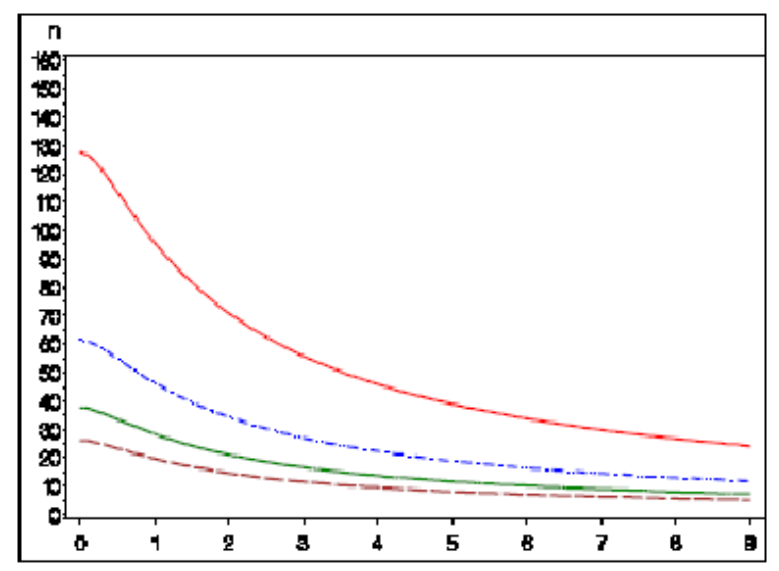

Fig. 2: The plot of the sample size $n$ versus $\xi^{2}$ value for $C_{A Q L}=$ $0.8264,0.6944,0.5917,0.5102,0.4444, C_{L T P D}=1.0$ with $\alpha=$ $0.05, \beta=0.10$ (from bottom to top in plot).

follows

Step 1Choose the producer's risk and consumer's risk. Select the process capability requirements $\left(C_{A Q L}, C_{L T P D}\right)$ at two risks.

Step 2 Take a random sample of size $n(\geq 1)$ and compute $\hat{C}_{p p}$

Step 3 Make a decision on the lot as follows:

1) Accept the lot if $\hat{C}_{p p}<c$

2) Otherwise, reject the lot
Table 2: $(\mathrm{n}, \mathrm{c})$ values for $\alpha$-risk $=0.01,0.025(0.025) 0.10, \beta$ risk $=0.01,0.025(0.025) 0.10$ with various $\left(C_{A Q L}, C_{L T P D}\right)$

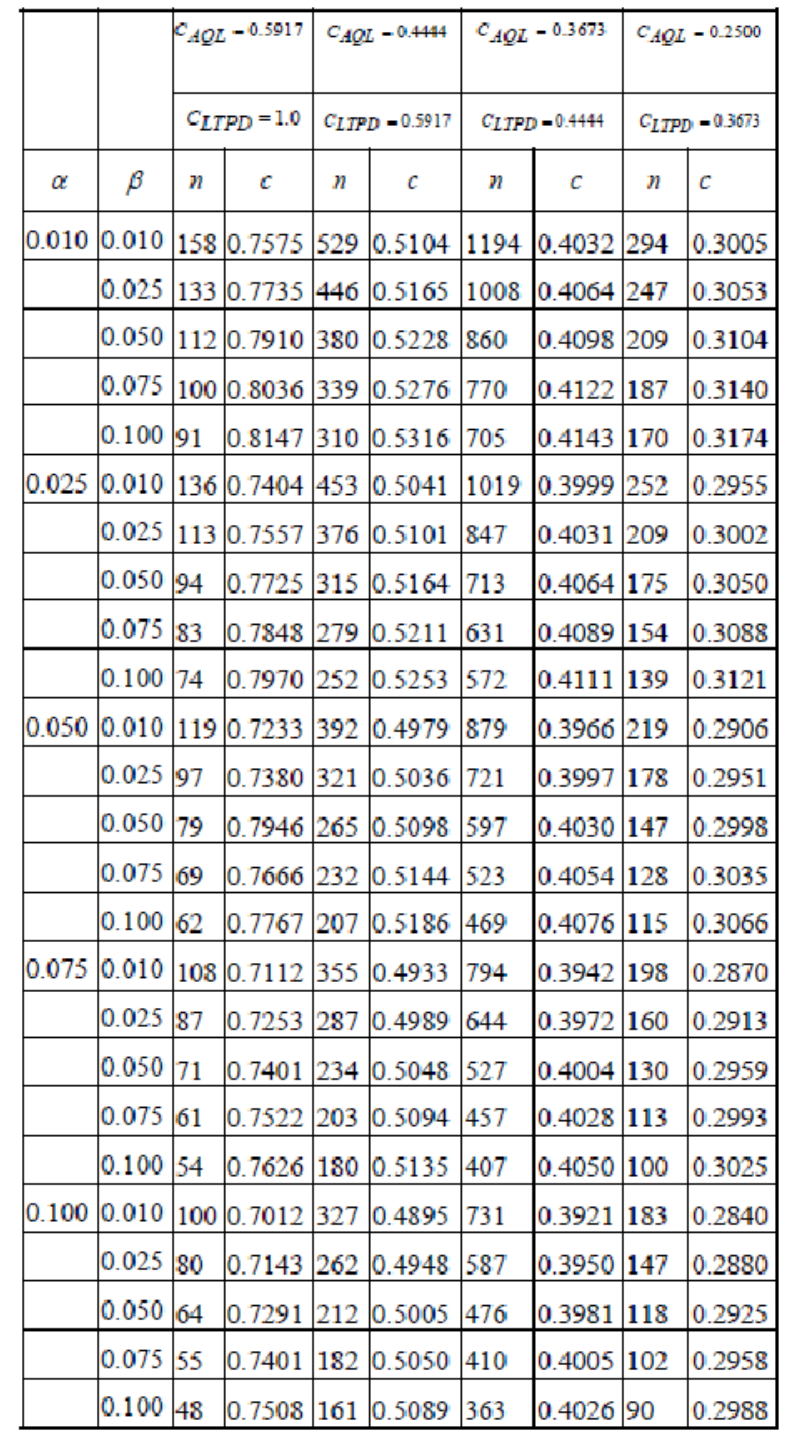

\section{An Illustrative Example}

To show the applicability of the proposed methodology, we present a case taken from Yen and Chang (2009) for example.For this amplified pressure sensor process, the Span is the focused characteristic. The specification limits are $T=2.0 \mathrm{~V}, U S L=2.1 \mathrm{~V}$, and $L S L=1.9 \mathrm{~V}$. Suppose the contract formulated from the supplier and the consumer, assume the values of $C_{A Q L}$ and $C_{L T P D}$ are set to 0.5917 and 1.0 with the $\alpha=0.025$ and $\beta=0.01$, respectively. Based on the above specified values in the contract, we could find the critical acceptance value and inspected sample size of the sampling plan $(n, c)=(136$, 0.7404 ) from the Table 2 . Hence, the inspected samples are taken from the lot randomly and the observed measurements are displayed in Table 3 The normal 


\section{Normal P-P Plot of Span}

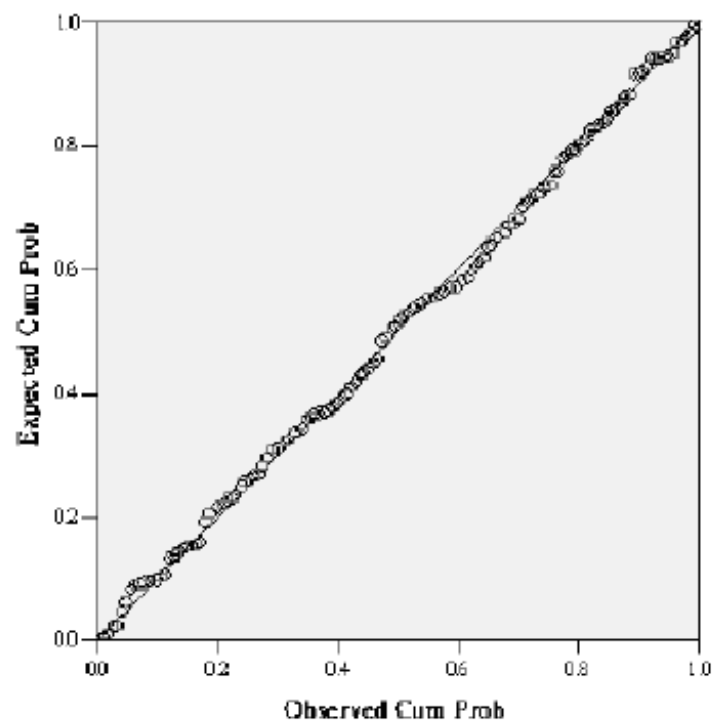

Fig. 3: The normal probability plot for the sample data

probability plot is depicted in Figure 3, which implies the sample data is from normal distribution.

The 136 observations from a sample are displayed in Table 3 The normal probability plot is depicted in Figure 3, which implies the sample data is from normal distribution. The Kolmogorov-Smirnov test is applied to further justify this assumption. Based on these inspections, we obtain that

$$
\bar{X}=1.9806, S_{n}^{2}=0.00036,
$$

and

$$
\widehat{C}_{p p}=\frac{(\bar{X}-T)^{2}}{D^{2}}+\frac{S_{n}^{2}}{D^{2}}=0.6627
$$

Therefore, in this study, the lot will be accepted by the consumer, since the sample estimated value 0.6627 is smaller than the critical acceptance value 0.7404 of the sampling plan significantly. Therefore, the buyer should accept the lot.

\section{Conclusion}

The traditional variable acceptance sampling plans do not consider the viewpoint that the quality between products falling within the specification limits may be not equal. In this paper, we developed a variable acceptance sampling plan based on incapability index $C_{p p}$ to deal with lot sentencing with process loss consideration. We tabulated the required sample size $n$ and the critical acceptance value $c$ for various $\alpha, \beta$, and the corresponding acceptable quality levels. Finally, one example is taken to
Table 3: The data for amplified pressure sensors

\begin{tabular}{llllllll}
\hline 1.9422 & 1.9651 & 2.0230 & 1.9712 & 1.9975 & 2.0164 & 1.9927 & 1.9566 \\
1.9738 & 1.9541 & 1.9800 & 1.9596 & 1.9811 & 2.0088 & 1.9858 & 1.9677 \\
2.0001 & 1.9659 & 1.9955 & 1.9842 & 1.9909 & 1.9829 & 1.9684 & 1.9942 \\
1.9897 & 1.9836 & 1.9891 & 1.9608 & 2.0109 & 1.9912 & 2.0077 & 1.9803 \\
2.0106 & 1.9885 & 1.9704 & 1.9882 & 1.9689 & 1.9553 & 1.9741 & 1.9825 \\
1.9640 & 2.0187 & 1.9616 & 1.9865 & 1.9556 & 1.9817 & 1.9774 & 1.9316 \\
1.9841 & 1.9919 & 1.9737 & 1.9958 & 2.0121 & 2.0021 & 1.9665 & 1.9773 \\
1.9841 & 1.9570 & 1.9610 & 2.0015 & 1.9750 & 1.9825 & 1.9758 & 1.9682 \\
1.9668 & 1.9696 & 2.0334 & 1.9656 & 1.9819 & 2.0116 & 1.9754 & 1.9986 \\
2.0114 & 1.9861 & 1.9743 & 1.9594 & 1.9712 & 1.9849 & 1.9711 & 1.9486 \\
1.9837 & 1.9424 & 1.9744 & 1.9605 & 1.9719 & 1.9656 & 1.9549 & 2.0174 \\
1.9779 & 2.0072 & 1.9875 & 1.9781 & 1.9834 & 1.9893 & 1.9276 & 1.9513 \\
1.9971 & 1.9963 & 1.9375 & 1.9941 & 1.9763 & 2.0108 & 1.9687 & 1.9559 \\
1.9611 & 1.9729 & 1.9992 & 1.9925 & 2.0073 & 1.9742 & 1.9557 & 1.9726 \\
1.9964 & 1.9614 & 1.9768 & 1.9991 & 1.9832 & 1.9847 & 1.9849 & 1.9918 \\
1.9748 & 1.9664 & 2.0035 & 1.9822 & 1.9882 & 1.9809 & 1.9920 & 1.9994 \\
2.0030 & 1.9786 & 1.9720 & 1.9834 & 1.9726 & 2.0012 & 1.9557 & 1.9874 \\
\hline
\end{tabular}

demonstrate our proposed approach.The proposed sampling plan provides the alternative for implementing the acceptance sampling plan.

\section{References}

[1] L. K. Chan, S. W. Cheng and F. A. Spiring, A new measure of process capability: Cpm. Journal of Quality Technology, 20, 162-175 (1988).

[2] N. G. Dasand S. K. Mitra, The effect of non-normality on sampling inspection. Sankhya, 26A, 169-176 (1964).

[3] K. Govindaraju and V. Soundararajan, Selection of single sampling plans for variables matching the MIL-STD-105 scheme. Journal of Quality Technology, 18, 234-238 (1986).

[4] M. Greenwichand B. L. Jahr-Schaffrath, A process incapability index. International Journal of Quality and Raliability Management, 12, 58-71 (1995).

[5] W. C. Guenther,Use of the binomial, hypergeometric, and Poisson tables to obtain sampling plans. Journal of Quality Technology, 1, 105-109 (1969).

[6] W. A. Hailey, Minimum sample size single sampling plans: a computerized approach. Journal of Quality Technology, 12, 230-235 (1980).

[7] A. Hald,Statistical Theory of Sampling Inspection by Attributes, Academic Press Inc., London (1981).

[8] W. J. Jennettand B. L. Welch, The control of proportion defective as judged by a single quality characteristic varying on a continuous scale. Journal of the Royal Statistical Society, Series B, 6, 80-88 (1939).

[9] V. E. Kane, Process capability indices. Journal of Quality Technology, 18, 41-52 (1986).

[10] J. H. K. Kao,MIL-STD-414: Sampling procedures and tables for inspection by variables for percent defective. Journal of Quality Technology, 3, 28-37 (1971).

[11] G. J. Liebermanand G. J. Resnikoff, Sampling plans for inspection by variables. Journal of the American Statistical Association, 50, 457-516 (1955).

[12] D. B. Owen, Variables sampling plans based on the normal distribution. Technometrics, 9, 417-423 (1967).

[13] L.J. Stephens, A closed form solution for single sample acceptance sampling plans. Journal of Quality Technology, 10, 159-163 (1978). 
[14] R. P. Sureshand T. V. Ramanathan, Acceptance sampling plans by variables for a class of symmetric distributions. Communications in Statistics: Simulation and Computation, 26, 1379-1391 (1997).

[15] W. L. Pearn and C. W. Wu, Critical acceptance values and sample sizes of a variables sampling plan for very low fraction of defectives. Omega - International Journal of Management Science, 34, 90-101 (2006).

[16] W. L. Pearn and C. W. Wu, Variables sampling plans with PPM fraction of defectives and process loss consideration. Journal of the Operational Research Society, 57, 450-459 (2006).

[17] W. L. Pearn and C. W. Wu, An effective decision making method for product acceptance. Omega - International Journal of Management Science, 35, 12-21 (2007).

[18] C. W. Wu and W. L. Pearn, A variables sampling plan based on Cpmk for product acceptance determination. European Journal of Operational Research, 184, 549-560 (2008).

[19] C. H. Yen and C. H. Chang, Designing Variables Sampling Plans with Process Loss Consideration. Communications in Statistics: Simulation and Computation, 38, 1579-1591 (2009).

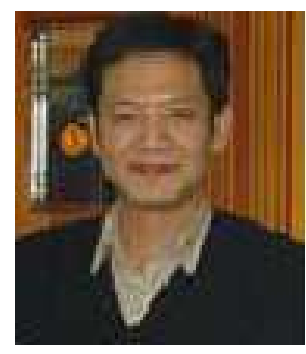

Liang-Chyau Sheu has a $\mathrm{Ph} . \mathrm{D}$. degree in Industrial Engineering of TsingHua University. $\mathrm{He}$ is currently a Associate Professor in Chung Hua Univeristy. His Research Interests is MIS, programming language, software system, Database Management System, Operations Research,

Algorithms.

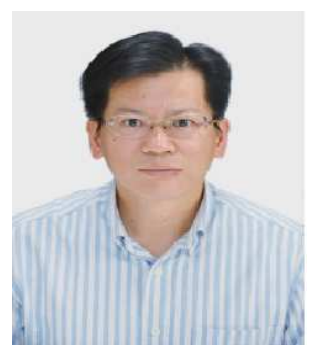

Ching-Ho Yen, has

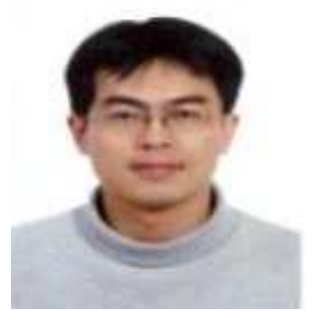

Management.

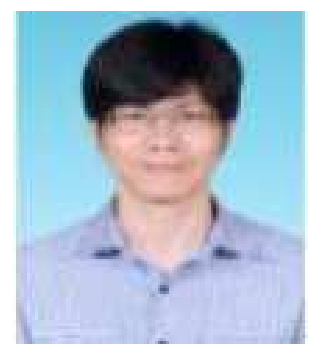
Analysis and programming SAS/R language.
Chia-Hao Chang has a Ph.D. degree in Statistics of North Dakota State University. $\mathrm{He}$ is currently an Associate Professor in Chang Gung University of Science and Technology, Chiayi Campus. His Research Interests is Nonparametric Statistics, Experimental Design, Categorical Data 